\title{
Dynamics of Carrier Injection in Picene Thin-Film Field-effect Transistors with an Ionic Liquid Sheet and Ionic Liquid Gel
}

\author{
Yuya Nagasaki ${ }^{\mathrm{a}}$, Ji-Hyun Lee ${ }^{\mathrm{a}}$, Yoshihiro Kubozono ${ }^{\mathrm{b}, \mathrm{c}}$ and Takashi Kambe ${ }^{\mathrm{a}, *}$ \\ a Department of Physics, Okayama University, Okayama 700-8530, Japan \\ ${ }^{\mathrm{b}}$ Research Laboratory for Interface Science, Okayama University, Okayama 700-8530, Japan \\ ${ }^{\mathrm{c}}$ Research Center of New Functional Materials for Energy Production, Storage and \\ Transport, Okayama University, Okayama 700-8530, Japan
}

*Corresponding author, Tel: +81-86-251-7829; fax: +81-86-251-7830,

E-mail address: kambe@science.okayama-u.ac.jp (T. Kambe)

Keywords: picene, electric double layer, field-effect transistor, electron spin resonance, ionic liquid gel

We fabricated picene thin-film field-effect transistors (FETs) with an ionic liquid gel and ionic liquid sheet as the gate electrolyte, and then used electron spin resonance (ESR) to investigate the carrier injection process in the organic electric double layer (EDL) FET. The ESR spectra strongly depended on the morphology of gate electrolytes. Three types of carrier injection processes in the EDL-FET were observed by examining the applied-bias time, organic-layer thickness, and gate-voltage dependencies of the electric-field-induced ESR spectrum: (1) interface injection due to electrostatic EDL formation, (2) bulk injection due to penetration of ions (electrochemical bulk doping), and (3) electrochemical reaction. These findings are significant for designing novel materials using the EDL-FET technique because three different carrier injection processes may lead to different physical properties, even in the same organic material.

\section{Introduction}

An electric double layer field-effect transistor (EDL-FET) is a powerful tool for investigating high-density carrier accumulation in various materials wherein only carriers are accumulated without any chemical doping of ions. ${ }^{[1-13]}$ Any additional factors, such as structural changes and the formation of scattering centers, may be ruled out in electrostatic carrier accumulation in an EDL capacitor. This allows us to selectively extract the intrinsic physical phenomena that are induced by carrier accumulation into materials. Recently, this technique has been effectively used with semiconductors ${ }^{[1-4]}$ and superconductors, ${ }^{[5]}$ revealing interesting physical properties. One of the first successful applications of an EDL-FET in solid state physics was the emergence of superconductivity in $\mathrm{SrTiO}_{3}{ }^{[1]}$ The superconducting transition in $\mathrm{SrTiO}_{3}$ 
was successfully achieved in an EDL-FET without doping any chemicals such as Nb. This is called field-induced superconductivity. Subsequently, superconductivity was produced by the EDL-FET technique in two-dimensional layered materials such as $\mathrm{ZrNCl}^{[2]} \mathrm{KTaO}_{3},{ }^{[3]}$ and $\mathrm{MoS}_{2}{ }^{[4]}$

These examples illustrate that the EDL-FET technique is a powerful way to realize novel physical properties in inorganic materials. However, no emergence of novel physical properties has yet been achieved in any organic materials. Although the EDL-FET technique has been applied to many organic materials, superconductivity was only observed in $\mathrm{C}_{60} \cdot{ }^{[7]} \mathrm{In}$ this case, electrochemical doping with electrolyte ions was required. This is intrinsically different from electrostatic carrier doping in which the material is not doped with any ion. We stress that electrostatic doping of carriers often coexists with electrochemical doping. ${ }^{[8]}$ In particular, the interface of thin films and single crystals of organic materials is so soft and flexible that electrolyte ions in electrolytes and ions in ionic liquids, used as EDL capacitors, can easily penetrate from the interface into bulk. Some studies have described electrochemical doping in EDL-FETs with organic materials ${ }^{[9,10]}$ and polymers. ${ }^{[8,11]}$ In such cases, electrochemical doping means reversible ion penetration into the bulk region of the active layer. We previously reported electrochemical carrier doping on organic thin films in an EDLFET. ${ }^{[12]}$ In this case, the EDL-FET with [7]phenacene thin films was fully investigated with an ionic liquid gel (ILG) for an EDL capacitor; this required an extremely long duration (more than $10^{3} \mathrm{~s}$ ) for saturation of the drain current, $I_{\mathrm{d}}$, measured at the fixed gate voltage, $V_{\mathrm{g}}$, and drain-source voltage, $V_{\mathrm{ds}}$. The enhancement of $I_{\mathrm{d}}$ with thickness of the [7]phenacene thin film verifies electrochemical carrier doping (or penetration of ions into the thin film). Thus, electrochemical doping is more significant in organic materials than in inorganic materials with hard interfaces, although it was recently found that electrochemical bulk doping occurs even in inorganic materials. ${ }^{[13]}$ Thus, a detailed investigation of the processes of electrostatic interface carrier doping and electrochemical bulk doping in organic materials is essential to determine how the carriers are accumulated and to be able to distinguish purely electrostatic interface doping. If carrier injection processes, such as electrostatic and electrochemical doping, can be precisely controlled using the EDL-FET technique, we may be able to exploit diverse functionalities in target materials.

In this study, we have fully investigated the process of gate-induced carrier accumulation in an organic thin film using electron spin resonance (ESR). The target organic material was picene, which consists of five benzene rings and is $\mathrm{W}$-shaped (phenacene-type structure). ESR studies for organic FETs with solid gate insulators ${ }^{[14,15,16]}$ and with ILGs 
using rubrene ${ }^{[17]}$ and poly(3-hexylthiophene $)^{[18]}$ have been reported. These studies mainly consider trap states in organic thin films and single-crystal FETs, whereas the present study addresses the process of carrier accumulation in an organic thin-film FET with an ionic liquid capacitor. The alkali-metal intercalated picene has shown superconducting transitions at 7 and $18 \mathrm{~K},{ }^{[19]}$ i.e., a high-density electron accumulation can produce superconductivity. We may obtain a strategy for field-induced superconductivity in picene by fully investigating the processes of electrostatic (interface) and electrochemical (bulk) carrier accumulation. Here, we used two types of EDL capacitors, i.e., an ILG and ionic liquid sheet (ILS) because two EDL capacitors may produce a clear difference in the process of carrier accumulation.

\section{Experimental}

We fabricated a picene thin film on a gold substrate by physical vapor deposition under vacuum. The typical dimensions of the device used in ESR experiments were $2(\mathrm{~mm}) \times 15(\mathrm{~mm}) \times d(\mu \mathrm{m})$, where the thickness was either $d=0.5 \mu \mathrm{m}$ or $1 \mu \mathrm{m}$. X-ray diffraction (XRD) patterns were measured with Smart Lab-Pro (RIGAKU) to investigate sample morphology and alignment of picene molecules. Sample morphology was also checked by atomic force microscopy experiments (SPA 400-DFM, SII Nano Technologies). The thin-film sample on the gold substrate and a Pt electrode were placed together in a quartz glass tube, which was filled with an ILG of $[\mathrm{bmim}] \mathrm{PF}_{6}$ as the gate electrolyte (see Figure 1(a)). We also measured ESR with an ILS as the gate electrolyte. The ILS was made by mixing a polymer (p[VDF-HFP], Sigma Aldrich, CO. Ltd.) and the ionic liquid. The sandwiched device with the Pt/ILS/picene/Au structure was placed in a quartz glass tube (see Figure 3(a)). We carefully checked the quality factor of the cavity to ensure that it was not reduced by insertion of the ILG and device. We measured frequency-dependent dielectric properties for the ILG and ILS at room temperature and obtained EDL capacitances for the ILG and ILS of $\sim 7 \mu \mathrm{F} / \mathrm{cm}^{2}$ and $\sim 1.2 \mu \mathrm{F} / \mathrm{cm}^{2}$, respectively. For both the ILS and ILG gate electrolytes, spin carriers can be injected by applying a negative voltage to the Pt electrode, implying that holes are injected into picene molecules. The number of spins, Ns, can be estimated from the integrated intensity of ESR signals. Time-dependent ESR spectra were measured with an X-band ESR spectrometer (Bruker, ESP-300e). All ESR spectra in this study were recorded at room temperature.

\section{Results and discussion}


The device used for ESR measurements with the ILG is shown in Figure 1(a), and photos of the picene thin film before and after the experiments are shown in Figure 1(b). The device consisted of two electrodes: the gate electrode (Pt wire) applying $V_{\mathrm{g}}$ and counter electrode (Au substrate) grounded. The picene thin film on the Au substrate was always grounded, and both electrodes were immersed in the ILG (bmim[PF 6$])$, i.e., all the picene thin film was in the ILG.

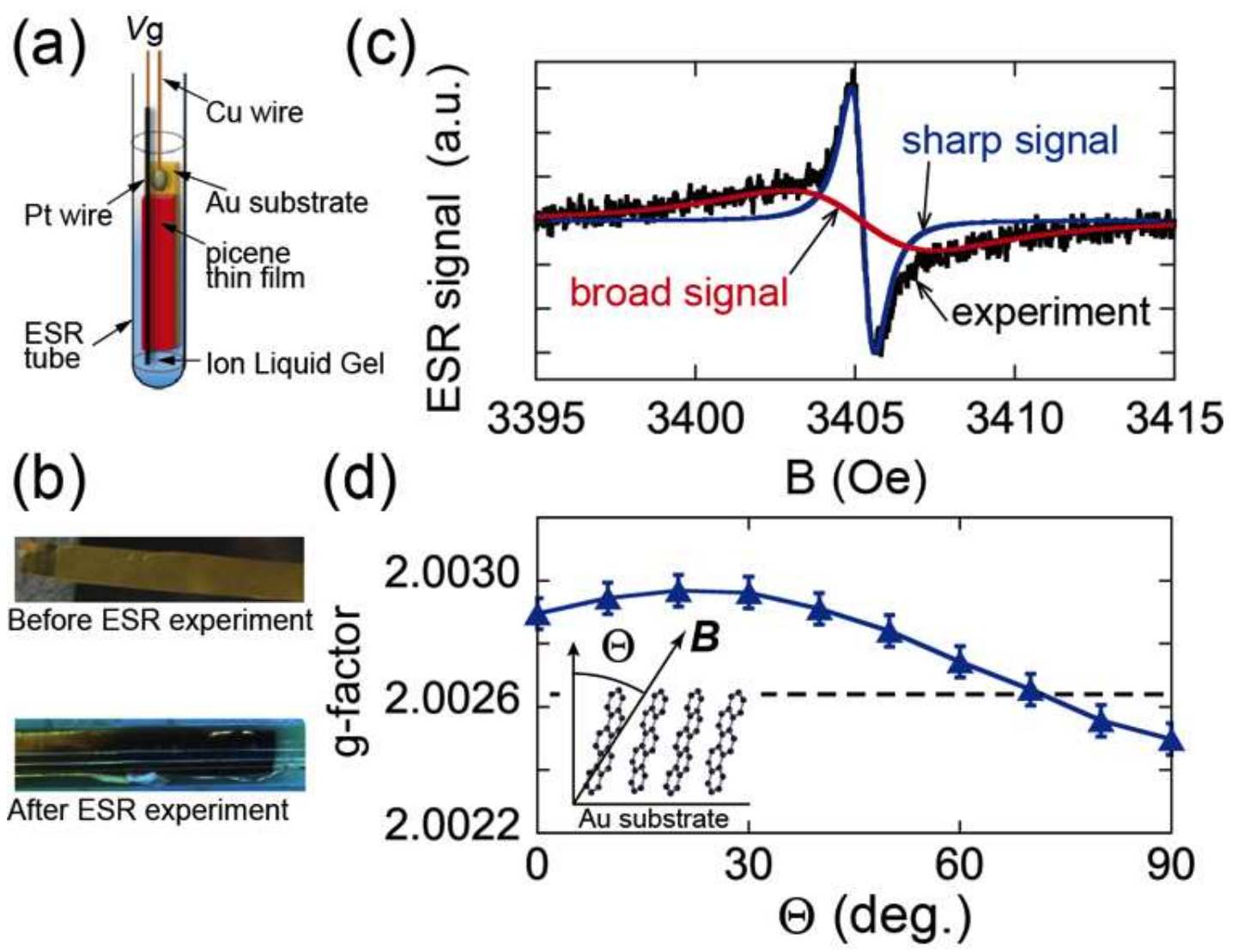

Figure 1. (a) Sketch of the ESR experimental setup for the picene thin-film FET using ILG. (b) Photos of the device before and after the ESR experiment. (c) Gate-induced ESR signals at $V_{\mathrm{g}}=-3 \mathrm{~V}$. (d) Angle dependence of $g$-factors for sharp ESR signals.

First, we present the ESR results using the ILG capacitor. Figure 1(c) shows the gate-induced ESR spectrum for a picene thin film of thickness $d=0.5 \mu \mathrm{m}$ to which a $V_{\mathrm{g}}$ of -3 $\mathrm{V}$ was applied. No ESR signals were observed at $V_{\mathrm{g}}=0 \mathrm{~V}$. The ESR spectrum (Figure 1(c)) observed at $V_{\mathrm{g}}=-3 \mathrm{~V}$ definitely decomposed into two components: one peak has a narrow line width $(\sim 0.5$ Oe: blue line), whereas the other has a broad line width ( 5 Oe: red line). Note that $V_{\mathrm{g}}=-3 \mathrm{~V}$ is slightly out of the electrochemical potential window for bmim[ $\left.\mathrm{PF}_{6}\right]$ at 
room temperature. ${ }^{[20]}$ When we applied a $V_{\mathrm{g}}$ within the electrochemical potential window, only a sharp ESR signal was observed.

Figure 1(d) shows the angle dependence of the $g$-factor for the sharp ESR signal at $V_{\mathrm{g}}=-3 \mathrm{~V}$. The error in the $g$-factor was \pm 0.00005 . The value $\Theta=0^{\circ}$ corresponds to the magnetic field, $\boldsymbol{B}$, perpendicular to the substrate. The $g$-factor for the sharp ESR signal clearly depends on the $\boldsymbol{B}$-direction and shows a large value when $\boldsymbol{B}$ is nearly perpendicular to the substrate. The XRD measurement for the picene thin film grown on the Au substrate indicates that the long molecular axis was inclined by $20-30^{\circ}$ to the normal axis of the substrate. ${ }^{[21]}$ Anisotropy of the $g$-factor for picene molecules has not been previously reported. The principal value of the $g$-factor along the long axis was the largest among the three principal values in rubrene, ${ }^{[17]}$ BEDT-TTF, ${ }^{[2]}$ and oligoacenes, ${ }^{[16]}$ whereas the corresponding $g$-factor was smallest in poly(3-hexylthiophene). ${ }^{[14]}$ Considering that the long axis of picene is aligned perpendicular to the Au substrate, ${ }^{[21]}$ the angle dependence of ESR for the sharp ESR signal clearly shows that the $g$-factor along the long molecular axis of picene is larger than those along other directions (see Figure 1(d)).

The dashed line in Figure 1(d) denotes the $g$-factor for picene anions in a THF solution, 2.00261 (see Figure S1). The average $g$-factor, 2.00273, evaluated from the angle dependence of the sharp signal is consistent with this value, verifying carrier injection into

(a)

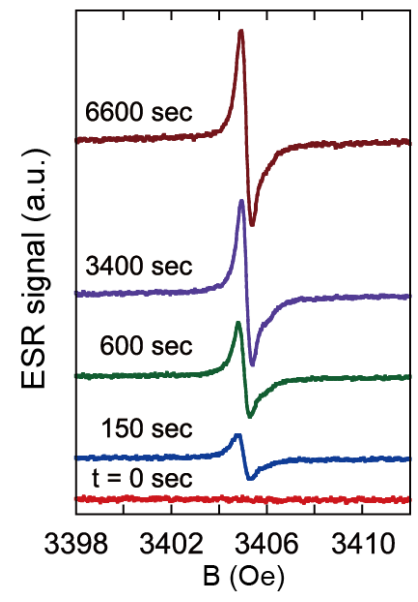

(b)
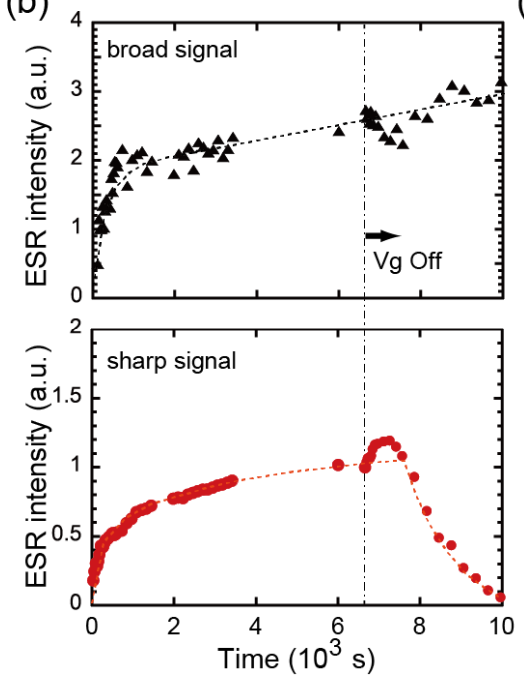

(c)
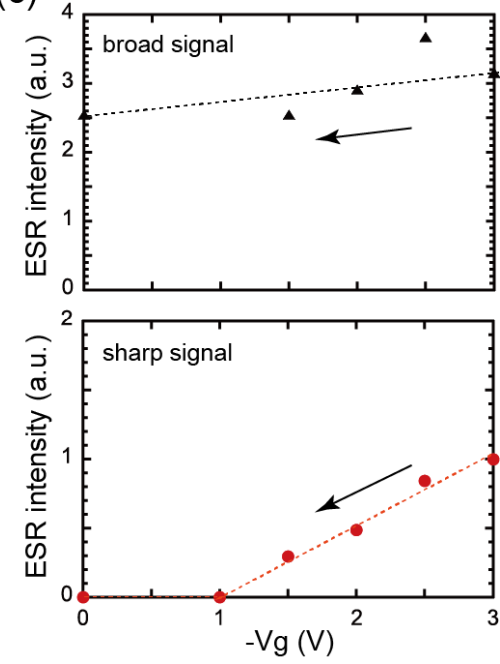

Figure 2. (a) Time dependence of ESR spectra at $V_{\mathrm{g}}=-3 \mathrm{~V}$ for the picene thin-film FET with ILG. The last $6600 \mathrm{~s}$ spectrum was recorded just before switching off the voltage. (b) Time dependence of ESR intensity. At about $6600 \mathrm{~s}, V_{\mathrm{g}}$ was switched off. Dotted lines are guides for the eye. (c) Gate dependence of ESR intensity. Closed triangles and closed circles (red) are results for broad and sharp ESR signals, respectively. 
picene molecules.

Figure 2(a) shows the time-evolution of an ESR spectrum measured at $V_{\mathrm{g}}=-3 \mathrm{~V}$. After $V_{\mathrm{g}}$ was applied, the ESR spectrum grew with time. Figure 2(b) summarizes the time dependencies of the integrated intensities of the sharp and broad ESR peaks; the time dependencies of the peak-to-peak line widths $\left.\left(\Delta B_{\mathrm{pp}}\right)\right)$ are shown in Figure S2. The ESR intensities of both components increased with time. After we confirmed that the ESR intensity of the sharp signal had saturated, the applied $V_{\mathrm{g}}$ was switched off at $\sim 6600$ s, i.e., $V_{\mathrm{g}}$ was changed from $-3 \mathrm{~V}$ to 0 , and the ESR signal was recorded. The intensity of the sharp signal decreased and vanished within $2000 \mathrm{~s}$ after $V_{\mathrm{g}}$ was switched off, whereas the intensity of the broad signal gradually increased even after $V_{\mathrm{g}}$ was switched off. When we applied $V_{\mathrm{g}}$ again, the sharp signal was restored, but the broad signal did not change as a function of time. Figure 2(c) shows the $V_{\mathrm{g}}$ dependence of ESR intensity. The intensity of the sharp signal linearly decreased with decreasing $V_{\mathrm{g}}$, whereas that of the broad signal did not. These results imply that the sharp signal was completely controlled by $V_{\mathrm{g}}$, whereas the broad signal was not. That is, the sharp ESR signal reflects $V_{\mathrm{g}}$-controllable and -reversible hole accumulation, whereas the broad ESR signal was due to an electrochemical reaction (irreversible), i.e., picene radicals were chemically produced. This often occurs in electrolyte-gated polymer transistors. ${ }^{[8]}$ In fact, $\Delta B_{\mathrm{pp}}$ of the broad ESR signal is close to that of the ESR signal for the picene anion in THF solution ( $~ 8 \mathrm{Oe})$, supporting the hypothesis that the broad signal is due to an electrochemical reaction. Furthermore, the color of the thin-film device changed to black after the ESR experiments (see Figure 1(b)), corroborating the electrochemical reaction.

To determine whether the sharp ESR signal originated from spin carriers accumulated only at the interface between the picene film and the ILG, the spin density, $N_{\mathrm{s}}$, was compared with the charge carrier density, $N_{\mathrm{c}}$; both $N_{\mathrm{s}}$ and $N_{\mathrm{c}}$ are given in $\mathrm{cm}^{-2}$. A value for $N_{\mathrm{c}}$ can be obtained using the simple expression, $N_{\mathrm{c}}=Q / e=C\left|V_{\mathrm{g}}\right| e$, where $Q, e$, and $C$ are the total number of charges, the elementary charge, and the capacitance per area of EDL, respectively. ${ }^{[23]}$ A value of $N_{\mathrm{c}}$ obtained from the capacitance refers only to the accumulated carrier density in the channel region of picene, i.e., the surface charge. If all spins detected in the sharp ESR spectrum correspond only to electrostatically accumulated holes (or surface holes), $N_{\mathrm{s}}$ must be equal to $N_{\mathrm{c}}$. However, $N_{\mathrm{s}}$ was larger than $N_{\mathrm{c}}$. Specifically, $N_{\mathrm{s}}$ and $N_{\mathrm{c}}$ at $V_{\mathrm{g}}=$ $-3 V$ were estimated to be $1.5 \times 10^{14}$ and $6.0 \times 10^{13} \mathrm{~cm}^{-2}$, respectively. This suggests the presence of additional spins other than electrostatically accumulated spins (or surface spins). This implies the penetration of holes (bulk hole accumulation) in the picene thin film in the case of the ILG capacitor. Thus, when ions can penetrate into the film, $N_{\mathrm{s}}$ is not equal to $N_{\mathrm{c}}$. In 
any case, the fact that $N_{\mathrm{s}}>N_{\mathrm{c}}$ indicates that spin carriers were generated not only at the interface but also in the bulk region.

The intensity of the sharp ESR signals increased with increasing thickness $d$. The ESR intensity per area for $d=1.0 \mu \mathrm{m}$ was about $30 \%$ stronger than that for $0.5 \mu \mathrm{m}$. If the entire picene film with $d=0.5 \mu \mathrm{m}$ is assumed to be uniformly doped, then approximately $0.11 \%$ of the picene molecules have spins, based on the total number of spins estimated from ESR and the total number of molecules. However, this assumption conflicts with the thickness dependence of the ESR intensity; the intensity is not proportional to the thickness, implying an inhomogeneous penetration of ions.

The thickness dependence of the ESR intensity suggests inhomogeneous penetration of ions into the bulk region of the picene film, and according to the electric-field decay, the density of penetrated ions decreased gradually from the interface into the bulk. A similar result was observed in the ESR on poly(3-hexylthiophene) $\mathrm{FET}^{[18]}$ in which the estimated high spin concentration could not be explained exclusively by interfacial accumulation. Transport experiments for [7]phenacene thin-film FETs also show this thickness dependence. ${ }^{[12]}$ Accordingly, spin carriers were injected not only at the interface but also in the bulk region of the picene layer, i.e., both electrochemical bulk and electrostatic interfacial

(a)

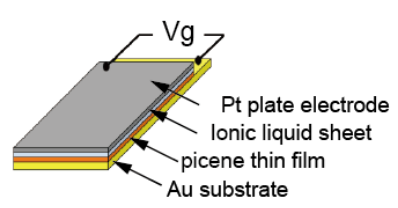

(d)

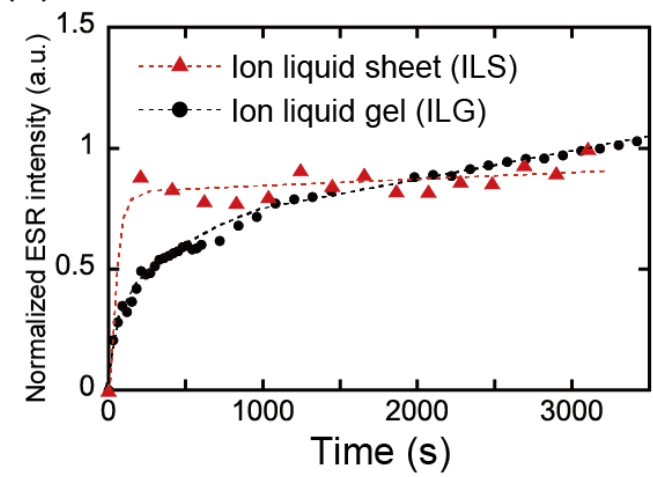

(b)

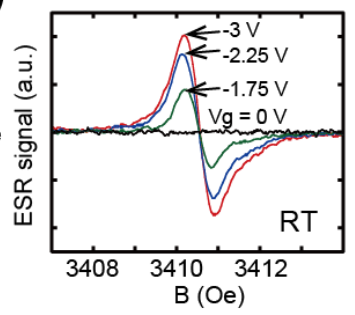

(c)

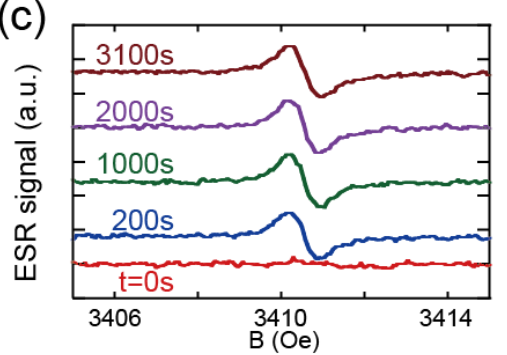

(e)

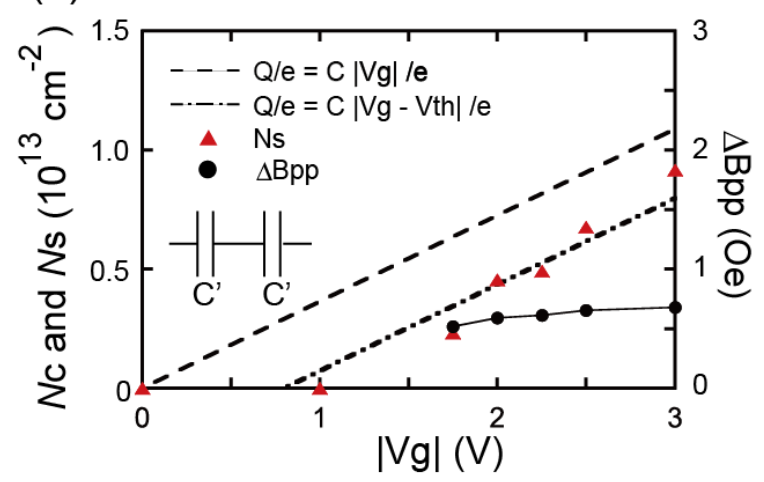

Figure 3. (a) Sketch of ESR experiment setup for the picene thin-film FET with the ILS. (b) Gate-induced ESR spectra at different $V_{\mathrm{g}}$. (c) Time dependence of the ESR spectra with the ILS at $V_{\mathrm{g}}=-3 \mathrm{~V}$. (d) Time dependence of ESR intensities for ILS and ILG. Dotted lines are guides for the eye. (e) $V_{\mathrm{g}}$ dependence of spin density and charge density per unit area (closed triangles) and $V_{\mathrm{s}}$ dependence of the ESR line width (closed circles). 
hole accumulations occurred in the picene FET with ILG.

The ESR experiments for the ILG capacitor imply the existence of three possible processes: two reversible processes (electrostatic interfacial and electrochemical bulk accumulations) extracted from the sharp signal and an irreversible process (electrochemical reaction) from the broad signal. The observed applied-bias time, organic-layer thickness, and gate-voltage dependencies for the sharp ESR signal rule out simple interfacial hole accumulation between the picene thin film and ILG. Electrochemical bulk hole accumulation occurs due to diffusion of charged molecules (or ions) through grain boundaries in the picene layer. This process is reversibly controlled by the applied $V_{\mathrm{g}}$ in the same manner as electrostatic interface hole accumulation.

Finally, we show ESR results with the ILS capacitor. ${ }^{[9]}$ The sandwiched device structure is depicted in Figure 3(a). Figure 3(b) shows the typical gate-induced ESR spectra with different $V_{\mathrm{g}}$ values for the picene thin film with $d=1.5 \mu \mathrm{m}$, where $\boldsymbol{B}$ was applied perpendicular to the substrate. No ESR signals were observed at $V_{\mathrm{g}}=0 \mathrm{~V}$. At $V_{\mathrm{g}}=-3 \mathrm{~V}$, an ESR spectrum with a single component was observed whose $\Delta B_{\mathrm{pp}}(\sim 0.4 \mathrm{Oe})$ was similar to the sharp signal for the ILG (Figure 1(c)). This ESR signal can be reversibly controlled by the applied $V_{\mathrm{g}}$. No broad signal was observed with $V_{\mathrm{g}}=-3 \mathrm{~V}$, suggesting no electrochemical reaction. Figures 3(c) and 3(d) show the time-evolution of the ESR spectrum and the time dependencies of the integrated intensity for the ILS together with the data for the ILG. The observed ESR grows within $100 \mathrm{~s}$ and shows a weak time dependence, which differs from the time dependence of the ILG. When we switched off the $V_{\mathrm{g}}$, the ESR signal rapidly disappeared within $20 \mathrm{~s}$. Moreover, the integrated ESR intensity for $d=1 \mu \mathrm{m}$ was almost the same as that for $d=0.5 \mu \mathrm{m}$, implying hole accumulation in a confined region of the thin film (electrostatic interfacial hole accumulation).

Figure 3(e) shows $N_{\mathrm{s}}$ estimated from the ESR intensity as a function of $V_{\mathrm{g}}$. The dotted line is $N_{\mathrm{c}}$ calculated from the equation described above. The dot-dash line corresponds to $N^{\prime}{ }_{\mathrm{c}}$ $=Q^{\prime} / e=C\left|V_{\mathrm{g}}-V_{\text {th }}\right| e$, where $V_{\text {th }}$ refers to the threshold voltage. As shown in Figure 3(e), $N_{\mathrm{s}}$ $\left(V_{\mathrm{g}}\right)$ is consistent with $N_{\mathrm{c}}^{\prime}\left(V_{\mathrm{g}}\right)$, establishing the occurrence of a purely interfacial injection. The value of $V_{\text {th }}$ obtained from the ESR $(\sim 0.8 \mathrm{~V})$ was almost consistent with that $(\sim 0.87 \mathrm{~V})$ evaluated from the thin-film picene FET with the ILS. ${ }^{[24]}$ Below $\left|V_{\mathrm{th}}\right|$, the injected carriers may not contribute ESR signals because holes doubly occupy a trap level. Therefore, it is reasonable to find consistency between $N_{c}^{\prime}$ and $N_{\mathrm{s}}$ only for interfacial hole accumulation. Thus, for ILS, we can rule out penetration of ions into the bulk region. That is, the organic interface was undamaged without penetration of ions into the bulk region. A similar 
discussion has been reported based on the $V_{\mathrm{g}}$ dependence of ESR line widths in rubrene single-crystal FET. ${ }^{[17]}$ Figure 3(e) also shows a plot of the ESR line width vs $V_{\mathrm{g}}$. The value of $\Delta B_{\mathrm{pp}}$ scarcely depends on $V_{\mathrm{g}}$ or on time (see Figure $2 \mathrm{~S}$ ); this suggests a few deep-trap sites because of the undamaged interface in the case of ILS. ${ }^{[17]}$ Using single-crystal picene should be more effective for reducing penetration of the ions.

\section{Conclusions}

Electric-field-induced ESR spectra were observed in a picene FET with ILG and ILS. Three different processes (interfacial and bulk hole-accumulations and electrochemical reaction) occurred for the ILG but only one process (interfacial hole accumulation) occurred for the ILS. We emphasize that there was pure electrostatic carrier doping at the interface with the ILS capacitor. The estimated value for $N_{\mathrm{s}}$ at $V_{\mathrm{g}}=-3 \mathrm{~V}$ corresponds to $\sim 0.03$ spins per one

picene molecule at the interface. Thus, a higher carrier accumulation will be necessary to manipulate the physical properties of picene. The present study addresses the accumulation process in a picene film. This is the first step in a comprehensive study addressing superconductivity in picene. This study may contribute to effective carrier accumulation in picene at low temperatures. Finally, we stress that the different types of carrier injection processes observed using ILG and ILS may enable us to exploit diverse functionalities in picene.

\section{Acknowledgements}

This research is partly supported by a Grant-in-Aid for Scientific Research from the Japanese Ministry of Education, Culture, Sports, Science and Technology, Japan (23340104 and 22244045) and by the LEMSUPER project (JST-EU Superconductor project) of the Japan Science and Technology Agency (JST).

\section{References}

[1] K. Ueno, S. Nakamura, H. Shimotani, A. Ohtomo, N. Kimura, T. Nojima, H. Aoki, Y. Iwasa, M. Kawasaki, Nat. Mater., 2008, 7, 855. 
[2] J. T. Ye, S. Inoue, K. Kobayashi, Y. Kasahara, H. T. Yuan, H. Shimotani, Y. Iwasa, Nat. Mater., 2010, 9, 125.

[3] K. Ueno, S. Namamura, H. Shimotani, H. T. Yuan, N. Kimura, T. Nojima, H. Aoki, Y. Iwasa, M. Kawasaki, Nat. Nano., 2011, 6, 408.

[4] J. Ye, Y. J. Zhang, R. Akashi, M. S. Bahramy, R. Arita, Y. Iwasa, Science, 2012, 338, 1193.

[5] A. T. Bollinger, G. Dubuis, J. Yoon, D. Pavuna, J. Misewich, I. Bozovic, Nature, 2011, 472,458 .

[6] A. S. Dhoot, S. C. Wimbush, T. Benseman, Adv. Mater., 2010, 22, 2529.

[7] Y. Kasahara, T. Nishijima, T. Sato, T. Takeuchi, J. T. Ye, H. T. Yuan, H. Shimotani, Y. Iwasa, J. Phys. Conf. Series, 2012, 400, 022049.

[8] J. D. Yuen, A. S. Dhoot, E. B. Namdas, N. E. Coates, M. Heeney, I. McCulloch, D. Moses, A. J. Heeger, J. Am. Chem. Soc., 2007, 129, 14367.

[9] K. H. Lee, M. S. Kang, S. Zhang, Y. Gu, T. P. Lodge, C. P. Frisbie, Adv. Mater., 2012, $24,4457$.

[10] M. Hamedi, L. Herlogsson, X. Crispin, R. Marcilla, Adv. Mater., 2009, 21, 573.

[11] J. Lee, L. G. Kaake, H. J. Cho, X.-Y. Zhu, T. P. Lodge, C. P. Frisbie, J. Phys. Chem. C, 2009, 113, 8972 .

[12] Y. Kaji, K. Ogawa, R. Eguchi, H. Goto, Y. Sugawara, T. Kambe, K. Akaike, S. Gohda, A. Fujiwara, Y. Kubozono, Org. Elecron., 2011, 12, 2076.

[13] T. Nojima, H. Tada, S. Nakamura, N. Kobayashi, H. Shimotani, Y. Iwasa, Phys. Rev. B, 2011, 84, 020502(R).

[14] M. Tsuji, N. Arai, K. Marumoto, J. Takeya, Y. Shimoi, H. Tanaka, S. Kuroda, T. Takenobu, Y. Iwasa, Appl. Phys. Express, 2011, 4, 085702.

[15] S. Watanabe, H. Tanaka, S. Kuroda, A. Toda, S. Nagano, T. Seki, A. Kimoto, J. Abe, Appl. Phys. Lett., 2010, 96, 173302.

[16] Y. Shimoi et al., Mol. Cryst. Liq. Cryst., 2012, 566, 33. 
[17] Y. Takahashi, M. Tsuji, T. Takenobu, Y. Iwasa, K. Marumoto, Appl. Phys. Express, 2013, 6, 041603.

[18] M. Tsuji, Y. Takahashi, Y. Sakurai, Y. Yomogida, T. Takenobu, Y. Iwasa, K. Marumoto, Appl. Phys. Lett., 2013, 102, 133301.

[19] R. Mitsuhashi, Y. Suzuki, Y. Yamanari, H. Mitamura, T. Kambe, N. Ikeda, H. Okamoto, A. Fujiwara, M. Yamaji, N. Kawasaki, Y. Maniwa, Y. Kubozono, Nature, 2010, 464, 76.

[20] V. M. Hultgren, A. W. Mariotti, A. M. Bond, A. G. Wedd, Anal. Chem., 2002, 74, 3151.

[21] H. Okamoto, R. Eguchi, S. Hamao, H. Goto, K. Gotoh, Y. Sakai, M. Izumi, Y. Takaguchi, S. Gohda, Y. Kubozono, Sci. Rep., 2014, 4, 5330.

[22] N. Kinoshita, M. Tokumoto, H. Anzai, G. Saito, J. Phys. Soc. Jpn., 1985, 54, 4498.

[23] K. Marumoto, Y. Muramatsu, Y. Nagano, T. Iwata, S. Ukai, H. Ito, S. Kuroda, Y. Shimoi, S. Abe, J. Phys. Soc. Jpn., 2005, 74, 3066.

[24] Y. Shimo, H. Goto, Y. Kubozono, Y. Hayashi, Unpublished work, 2014.

\section{Graphical abstract}

Dynamics of Carrier Injection in Picene Thin-Film Field-effect Transistors with an Ionic Liquid Sheet and Ionic Liquid Gel

Yuya Nagasaki, Ji-Hyun Lee, Yoshihiro Kubozono and Takashi Kambe*
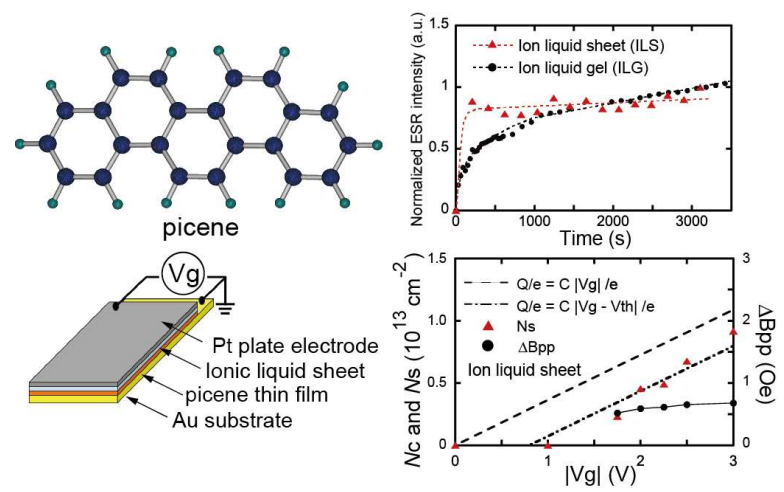